\title{
Quando Pintar Quadros Não É (Só) Atividade Artística: Construção da Escala de Interesses Profissionais para Pessoas com Deficiência Visual
}

\author{
Leonardo de Oliveira Barros ${ }^{1}$, Rodolfo. A. M. Ambiel \\ Universidade São Francisco, Campinas-SP, Brasil
}

\section{RESUMO}

O objetivo deste trabalho é apresentar a construção da Escala de Interesses Profissionais para pessoas com Deficiência Visual (EIDV) embasada na teoria de Holland e as evidências de validade com base no conteúdo e na estrutura interna. Inicialmente foram construídos 120 itens divididos igualmente entre as seis tipologias teóricas (realista, investigativo, artístico, social, empreendedor e convencional) e ocorreu a análise de juízes por doutoras em Psicologia e psicólogas de instituições de apoio à pessoa com deficiência visual, seguido do estudo piloto. Para o estudo de estrutura interna, participaram de 137 deficientes visuais, com idade a partir de 18 anos. Os dados foram analisados com estatísticas descritivas, análise fatorial exploratória e cálculo do alfa de Cronbach. Os resultados forneceram a confirmação empírica dos seis fatores teóricos e índices adequados de confiabilidade. Acredita-se que o instrumento contribua com a área de avaliação psicológica e para a identificação de interesses profissionais de deficientes visuais.

Palavras-chaves: avaliação psicológica; orientação profissional; deficiência visual; psicometria; construção do teste.

\begin{abstract}
When Painting Pictures Is Not (Just) Artistic Activity: Building the Professional Interests Scale for People with Visual Impairment

The objective of this study is to present the construction of a Professional Interests Scale for People with Visual Impairment (PISVI) based on the Holland's theory, and validity evidence based on content and internal structure. Initially, 120 items were divided equally among the six theoretical typologies (realistic, investigative, artistic, social, entrepreneur, and conventional), analyzed by judges with PhDs in Psychology and psychologists in support institutions for persons with visual impairment, then followed by a Pilot Study. In studying the internal structure, 137 visually impaired persons over age 18 participated. Data were analyzed with descriptive statistics, exploratory factor analysis and Cronbach's alpha calculation. Results provided empirical confirmation of the six theoretical factors and adequate reliability indexes. It is believed that the instrument contributes to the area of psychological assessment and to the identification of professional interests of the visually impaired.
\end{abstract}

Keywords: psychological evaluation; professional orientation; visual impairment; psychometry; test construction.

\section{RESUMEN - Cuando Pintar Cuadros No Es (Sólo) Actividad Artística: Construcción de la Escala de Intereses Profesionales para Personas con Deficiencia Visual}

El objetivo de este trabajo fue presentar la construcción de la Escala de Intereses Profesionales para Personas con deficiencia visual, basado en la teoría de Holland y evidencias de validez basadas en el contenido y estructura interna. Inicialmente, fueron construídos 120 ítems divididos en partes iguales entre las seis tipologías teóricas (realista, investigador, artístico, social, emprendedor y convencional) y se llevó a cabo el análisis de jueces por doctores en Psicología y por psicólogos de instituciones de apoyo a personas con deficiencia visual, seguido del Estudio Piloto. Para el estudio de estructura interna, participaron 137 personas con deficiencia visual, a partir de 18 años. Los datos se analizaron con estadísticas descriptivas, análisis factorial exploratorio y cálculo de alfa de Cronbach. Los resultados proporcionaron la confirmación empírica de los seis factores teóricos e índices adecuados de confiabilidad. El instrumento contribuirá en el área de evaluación psicológica y en la identificación de intereses profesionales de personas con deficiencia visual.

Palabras clave: evaluación psicológica; orientación professional; deficiencia visual; psicometría; construcción del test.

A deficiência, de acordo com a World Health Organization (WHO), é "complexa, dinâmica, multidimensional e questionável” (WHO, 2012, p. 4), devendo ser entendida a partir da interação entre problemas de saúde e fatores contextuais, pessoais e ambientais. Para a Classificação Internacional de Funcionalidade, Incapacidade e Saúde (CIF - WHO, 2001), é necessário compreender a deficiência como um modelo biopsicossocial, tornando possível retirar a deficiência como atributo exclusivo da pessoa e integrar as barreiras impostas socialmente (Barnes, 2012).

Especificamente sobre a deficiência visual, estimativas da WHO (2010) apontam que há no mundo cerca de 314 milhões de pessoas por ela acometida, sendo 269 
milhões com visão subnormal e 45 milhões com cegueira. No Brasil, o Censo Demográfico realizado pelo Instituto Brasileiro de Geografia e Estatística (IBGE), em 2010, contabilizou que, dos 45,5 milhões de brasileiros que autodeclararam ter alguma deficiência, 35,7 milhões são deficientes visuais ( $18,8 \%$ da população). Ainda que sem separação por tipo de deficiência, os relatórios mostram maior concentração de pessoas com alguma deficiência na região Nordeste, taxa de alfabetização e nível de escolaridade menor para pessoas desse grupo quando comparados com a população geral e que, para a colocação no mercado de trabalho, a deficiência é mais limitante para homens do que para mulheres (IBGE, 2010).

No campo do trabalho, a Orientação Profissional pode contribuir para que a inclusão da pessoa com deficiência ocorra, uma vez que se propõe a cooperar na construção de projetos de vida laboral (Salgado, 2002), sem negar a existência de fatores psíquicos, físicos e sociais limitantes, porém, não vendo nessas limitações um impedimento a inserção nas atividades sociais como o trabalho (Ribeiro, 2014). A Orientação Profissional é entendida como um processo de facilitação à escolha $\mathrm{e}$ construção da trajetória profissional e tem em seus objetivos compreender as variáveis que interferem na tomada de decisão, podendo fazer uso de instrumentos para mensuração das variáveis envolvidas, tais como habilidades, traços de personalidade e interesses (Savickas, 2015). Em relação aos instrumentos utilizados nos processos, os instrumentos de interesses profissionais são os mais frequentes (Ambiel \& Polli, 2011), fato relacionado a importância do construto que é tido como central para a área (Ambiel, Lamas, \& Melo-Silva, 2017).

Dentre os instrumentos de interesses profissionais, há um destaque no cenário nacional e internacional para aqueles que foram construídos a partir da Teoria de Personalidade Vocacional e Ambientes de Trabalhos (Holland, 1985). Nessa perspectiva, entende-se que há seis padrões de personalidade e consequentemente de interesses, sendo as tipologias Realista, Investigativo, Artístico, Social, Empreendedor e Convencional. Na literatura, encontram-se diversos instrumentos embasados na teoria com populações sem deficiência e distintas em relação à idade e nacionalidade (Holland, Fritzsche, \& Powell, 1994; Mansão \& Noronha, 2011; Primi, Mansão, Muniz, \& Nunes, 2009; Sampaio, 2013; Teixeira, Castro, \& Cavalheiro, 2008). Porém, não são encontrados instrumentos padronizados para a população com deficiência e, em especial, com deficientes visuais, fato que restringe a prática avaliativa e a intervenção de orientadores profissionais com esse público (Barros \& Murgo, 2014).

A compreensão das especificidades no desenvolvimento psíquico de pessoas com deficiência visual é importante para os psicólogos e pesquisadores em Psicologia, para que sejam desenvolvimentos instrumentos e estratégias de atendimento adequadas para a população. No tocante à prática de avaliação psicológica de pessoas com deficiência, a American Psychological Association (APA, 2011) lançou a Guidelines for Assessment of and Intervention with Persons with Desabilities orientando que, nesse serviço, os psicólogos devem considerar a deficiência como dimensão da diversidade e em conjunto com outros aspectos individuais e contextuais. Os profissionais são instruídos a utilizar uma abordagem de avaliação psicometricamente justa, compreensiva e apropriada para clientes com deficiência e devem também garantir que a acessibilidade e equidade ocorram nos ambientes profissionais, incluindo os instrumentos utilizados e o contexto em que a avaliação ocorre.

Em relação às práticas avaliativas utilizadas com pessoas com deficiência visual, esta não se limita apenas ao uso de instrumentos psicométricos, podendo ocorrer com o uso de instrumentos qualitativos para avaliar componentes sociais, educacionais, ocupacionais, saúde, psicomotores, entre outros (Serra et al., 2013; Rebouças, Araújo, Braga, Fernandes, \& Costa, 2016). Todavia, instrumentos psicométricos construídos ou adaptados para pessoas com deficiência visual podem ser uma contribuição ao trabalho dos psicólogos com a população em questão, de modo a permitir que utilizem medidas adequadas para mensurar atributos e delinear intervenções, respeitando os parâmetros éticos da profissão e as especificidades decorrentes da deficiência visual.

Nessa direção, entendendo que a Psicologia no Brasil tem definido em seu Código de Ética como princípios fundamentais a promoção da igualdade, o combate à discriminação e o compromisso com a universalização do acesso às informações, aos serviços e ao conhecimento da ciência psicológica, torna-se necessário que a área de avaliação psicológica consiga contemplar todos os públicos que dela possam fazer uso. Todavia, a construção ou adaptação de instrumentos para pessoas com deficiência visual no cenário nacional ainda é escassa e têm surgido como tema de estudos nos últimos anos, utilizando formatos e faixas etárias distintas na avaliação de construtos, como a inteligência (Campos \& Nakano, 2014; Lins, 2016), estresse infantil (Filgueiras et al., 2014) e personalidade (Oliveira, 2013). Tais estudos são importantes e permitem refletir sobre as possibilidades de avaliação psicológica com essa população e, além disso, a problematização da temática torna-se uma oportunidade de expandir o alcance e o impacto social do conhecimento produzido na área (Oliveira, 2013).

No intuito de contribuir para a área de avaliação psicológica de pessoas com deficiência visual e para o campo de Orientação Profissional, esta pesquisa tem por objetivo apresentar o processo de construção da Escala de Interesses Profissionais para pessoas com Deficiência Visual (EIDV), utilizando como embasamento teórico os pressupostos de Holland (1975/1985). Além disso, são relatados os primeiros estudos de propriedades psicométricas por meio da busca de evidências de validade 
com base no conteúdo e na estrutura interna. $\mathrm{O}$ estudo é apresentado em duas etapas, sendo a primeira referente aos procedimentos de construção e evidências de validade com base no conteúdo (elaboração de itens, análise de juízes e estudo piloto) e a segunda etapa com as evidências de validade baseada na estrutura interna.

\section{Construção da EIDV e evidências de validade baseadas no conteúdo}

Com o objetivo de subsidiar o processo de construção de itens, entre os meses de agosto a outubro de 2015, realizou-se o levantamento de profissões e ocupações nas quais pessoas com deficiência visual atuam. Foram consultados relatórios disponibilizados no Ministério do Trabalho e Emprego (MTE) e Classificação Brasileira de Ocupações (CBO), sites de instituições que atuam com deficientes visuais, memorandos de Instituições de Ensino Superior (IES) que continham histórico de cursos com alunos deficientes visuais matriculados e consulta à literatura científica. Desse modo, chegou-se a uma lista com 198 atividades profissionais, sendo que, após análise e exclusão de repetições, o número passou para 166.

A partir do levantamento realizado, as profissões e ocupações foram categorizadas de acordo com as tipologias de Holland (1975). Na sequência buscou-se a descrição e detalhamento das atividades referentes às profissões e ocupações encontradas. Com a descrição, foram elaborados os itens da escala, procurando integrar profissões e ocupações que demandassem diferentes níveis de formação e que pudessem ser realizadas tanto por pessoas com cegueira quanto por pessoas com baixa visão. Nesse sentido, foram construídos 20 itens por tipologia, totalizando 120.

Em seguida, houve avaliação de juízas, sendo que, para a avaliação de adequação teórica dos itens, participaram três pesquisadoras doutoras, com tempo médio de obtenção da última titulação de 5,3 anos, tendo como área de pesquisa Avaliação Psicológica, Orientação e Desenvolvimento de Carreira e com conhecimento da Teoria de Holland. Um segundo grupo de juízas foi escolhido para avaliação da possibilidade de execução da atividade por pessoas com deficiência visual, composto por três psicólogas de instituições de apoio à pessoa com deficiência visual do Estado de São Paulo, com tempo médio de atuação nas instituições de 8,3 anos.

Foram utilizando protocolos elaborados no Google Drive, contendo o Termo de Consentimento Livre e Esclarecido (TCLE), questionário de identificação e os 120 itens elaborados. As juízas pesquisadoras deveriam dizer se os itens pertenciam a tipologia para a qual haviam sido construídos, e as psicólogas avaliaram se a atividade descrita nos itens era possível de ser realizada apenas por pessoas com baixa visão, com cegueira, por ambos ou se não era possível de ser desempenhado por alguém com deficiência visual. Também solicitou que avaliassem a linguagem e apontassem profissões ou ocupações de deficientes visuais não contempladas nos itens.
A linguagem dos itens e a instrução de aplicação foi avaliada por $100 \%$ das juízas como adequadas, não apresentando problemas de compreensão. As juízas pesquisadoras sugeriram a exclusão de oito itens nas tipologias Realista, Investigativo e Empreendedor cada. Nos tipos Social e Convencional não houve concordância em quatro itens em cada tipologia. E, por último, a tipologia Artístico foi a única que teve apenas um item considerado inadequado do ponto de vista teórico. Totalizou-se, assim, a sugestão de exclusão de 33 itens.

A fim de selecionar os itens que atendessem ao mesmo tempo as demandas de pessoas com cegueira e baixa visão, optou-se por manter apenas os itens em que todas as juízas psicólogas avaliassem ser uma atividade possível de ser executada independentemente do tipo da deficiência visual e, assim, houve a sugestão de exclusão de 26 itens. Portanto, após o cruzamento de dados entre as análises das juízas pesquisadores e das psicólogas foram excluídos 48 itens. Nos tipos Realista, Investigativo e Convencional, foram excluídos 10 itens em cada. No tipo Empreendedor, foram excluídos nove itens; no Social, seis itens e, no Artístico, três itens. Assim, chegou-se a uma versão da Escala com 72 itens.

A versão resultante da análise de juízas foi submetida ao estudo piloto que aconteceu em duas formas, a fim de permitir que fosse testada a funcionalidade da plataforma on-line e a aplicabilidade em formato lápis e papel. Para o estudo piloto na modalidade lápis e papel, participaram cinco pessoas (três homens e duas mulheres) usuárias de uma instituição de apoio à pessoa com deficiência visual da cidade de São Paulo, com idades entre 34 e 70 anos $(M=56,6, D P=14,58)$, sendo três com baixa visão adquirida, um com baixa visão congênita e uma com cegueira adquirida. Os níveis de escolaridade variaram de ensino fundamental à ensino superior completo e haviam pessoas que nunca trabalharam, ativos no mercado de trabalho e aposentados. Já o estudo piloto em versão informatizada foi realizado por duas pessoas diferentes da amostra descrita acima, sendo uma do sexo feminino e outro do sexo masculino, com idades de 26 e 55 anos $(M=40,5)$, ex-usuários de instituições de apoio à pessoa com deficiência visual, residentes em cidades do interior de São Paulo, um com cegueira adquirida e ensino médio completo e outra com cegueira congênita, cursando ensino superior em Psicologia.

Inicialmente, houve o agendamento com uma das instituições e os participantes esclarecidos quanto aos objetivos da pesquisa e convidados para a coleta. A aplicação em formato lápis e papel ocorreu coletivamente, na qual o pesquisador leu os itens um por um e anotou as respostas dos participantes. Para a aplicação em formato informatizado, os participantes foram convidados por e-mail da rede de contatos do pesquisador, esclarecidos quanto aos objetivos da pesquisa e após aceitarem participar receberam o link de acesso ao protocolo. 
Os participantes que testaram o instrumento em formato informatizado, responderam uma vez por meio do celular com leitor de tela iOS 7, e uma segunda vez com computador, utilizando os leitores JAWS e NVDA. A única dificuldade encontrada foi ao final do formulário quando respondido com celular, pois o leitor não conseguia identificar o botão "Enviar formulário", levando a uma revisão no protocolo para que este enviasse automaticamente as respostas ao concluírem a última página. De modo concordante, os participantes apontaram não haver dificuldades em manusear a plataforma on-line, o que implicou em constatar que estava acessível para a população com deficiência visual com domínio dos recursos tecnológicos.

Sobre a possibilidade de execução das atividades propostas nos itens, apenas o item "Esculpir obras para uma exposição" foi avaliado como uma atividade que necessitava muito da visão, favorecendo pessoas com baixa visão. Houve dificuldade na compreensão de palavras mais complexas, como "vulnerabilidade", "lecionar" e "persuasão". Apenas um item não foi compreendido em sua totalidade pela participante com menor escolaridade (item: Conhecer as propriedades dos elementos da natureza).

Desse modo, considerando os apontamentos do estudo piloto, chegou-se à decisão de novas revisões e exclusões nos itens da escala. Dois itens do tipo Social que apresentaram dificuldade de compreensão foram excluídos, bem como o item "Esculpir obras para uma exposição" (Artístico) por ser considerada uma atividade mais favorável às pessoas com baixa visão. Outros dois itens das tipologias Realista e Empreendedor apresentaram dificuldade na compreensão, porém, com o intuito de se ter em média 10 itens por tipologia, tais itens foram revisados de modo a manter o mesmo sentido da ideia original.

\section{Evidências de validade baseadas na estrutura interna}

\section{Participantes}

Participaram dessa etapa 137 pessoas com deficiência visual, sendo 72 do sexo feminino (52,6\%) e 65 do sexo masculino $(47,4 \%)$, com idades variando entre 18 e 76 anos $(M=37,86, D P=14,39)$, distribuídos entre baixa visão congênita $(n=37,27 \%)$, baixa visão adquirida $(n=33,24,1 \%)$, cegueira congênita $(n=23,16,8 \%)$ e cegueira adquirida $(n=44,32,1 \%)$. Os participantes foram oriundos de todas as regiões do Brasil com respondentes em 15 estados, sendo a maior parte do estado de São Paulo $(n=101,73,7 \%)$, seguido dos estados de Santa Catarina $(n=8,5,8 \%)$, Rio Grande do Sul e Minas Gerais ( $n=6,4,4 \%$ em cada), Paraná $(n=4,2,9 \%)$, Bahia e Goiás ( $n=2,1,5 \%$ em cada). Nos estados de Sergipe, Brasília, Tocantins, Mato Grosso, Amapá, Paraíba, Rio de Janeiro, Espírito Santo houve a participação de uma pessoa em cada $(0,7 \%)$.
Em relação ao nível de escolaridade, uma menor parte declarou não ter concluído o ensino fundamental $(n=10,7,3 \%), 14,6 \%$ afirmou ter realizado apenas o ensino fundamental $(n=20), 48,9 \%$ da amostra declarou ter concluído o ensino médio $(n=67), 17,5 \%$ alegou ter concluído ensino superior $(n=24)$ e $11,7 \%$ informou ter pós-graduação $(n=16)$, variando entre os níveis lato sensu e stricto sensu. Sobre a situação laboral, 60 pessoas (43,8\%) encontram-se ativas no mercado de trabalho, 56 participantes não trabalham (40,9\%) e um menor grupo estava aposentado por tempo de serviço, invalidez ou benefício de prestação continuada $(n=21,5,3 \%)$.

\section{Instrumentos}

Questionário de Identificação. Composto por questões relativas à idade, sexo, grau e tipo da deficiência, escolaridade e atuações profissionais.

Escala de Interesses Profissionais para Deficientes Visuais (EIDV). Versão de 68 itens distribuídos nas tipologias realista (10 itens), investigativo (10 itens), artístico (16 itens), social (11 itens), empreendedor (11 itens) e convencional (10 itens). Os 68 itens foram respondidos em uma escala tipo Likert de cinco pontos, variando entre gostaria pouco ou nada (1) a gostaria muito (5) de realizar a atividade. A escala foi desenvolvida no Google Drive em blocos com 17 itens cada, sendo que, no início de cada página, havia a informação de quantos itens havia e as instruções de aplicação. O mesmo protocolo foi impresso para aplicação presencial.

\section{Procedimentos}

A pesquisa foi autorizada pelo Comitê de Ética em Pesquisa da Universidade São Francisco e, para responderem aos instrumentos, os participantes deveriam concordar com o Termo de Consentimento Livre e Esclarecido (TCLE). A coleta foi dividida entre a modalidade presencial em versão papel e informatizada utilizando o Google Docs. A recolha dos dados na modalidade presencial aconteceu em dias previamente agendado, entre os meses de abril e junho de 2016, em seis instituições de apoio à pessoa com deficiência visual do estado de São Paulo, sendo duas da capital e quatro do interior. A aplicação foi individual, sendo que o aplicador fez a leitura dos itens e registrou as respostas dos participantes, levando aproximadamente 10 minutos por pessoa.

A coleta em versão informatizada também ocorreu nos meses de abril a junho de 2016, sendo que o link da pesquisa foi divulgado em páginas e grupos específicos de pessoas com deficiência visual na rede social on-line Facebook. Ao total, a pesquisa foi divulgada em 33 grupos fechados e 14 páginas. Uma das instituições participantes forneceu um banco de dados com lista de e-mail de 50 pessoas que se voluntariaram anteriormente a outros projetos de pesquisa, sendo enviado o convite para participação no estudo. 
Dentre os participantes, 56 pessoas $(40,9 \%)$ responderam por meio de aplicação em versão papel com o aplicador lendo os itens e registrando as respostas. Contudo, a maior parte da amostra realizou a pesquisa de forma autônoma utilizando computador $(n=59,43,1 \%)$, aparelho celular $(n=20,14,6 \%)$ e Tablet $(n=2,1,5 \%)$. Em relação aos recursos de acessibilidade utilizados, 42 pessoas $(30,7 \%)$ utilizaram de leitores de tela sendo registrado o uso do Non Visual Desktop Access (NVDA), Vice Over, iOS, Jaws e leitor de tela de fábrica dos aparelhos celulares. O uso de fonte ampliada e lupa foi feito por 21 pessoas (15,3\%), 13 pessoas utilizaram computador com ajuda do aplicador (9,5\%) e cinco pessoas disseram que não houve a necessidade de usar nenhum recurso de acessibilidade $(3,6 \%)$.

\section{Análise de dados}

As análises foram realizadas com o software Statistical Package for the Social Sciences (SPSS) versão 21 e, para análise fatorial, utilizou-se o software Factor versão 10.5.02. Para a interpretação dos índices de ajustes da amostra para fatoração, foram considerados os critérios estabelecidos por Friel (2008). Para a extração de fatores seguiram-se os parâmetros de Dancey e Reid (2013), de Hair, Black, Babin, Anderson e Thatam (2006) e também foram empregados os apontamentos de Figueiredo-Filho e Silva-Junior (2010) para interpretar o índice de ajuste da amostra de Kaiser-Meyer-Olklin. A opção pelos métodos de extração e de rotação utilizados na análise fatorial exploratória seguiu as indicações de Damásio (2012), em que a fatoração por eixo principal é apontada como adequada para amostras com distribuição normal e a rotação oblimin é um método de rotação oblíqua simples e que apresenta bons estimadores para fatores que apresentam correlações entre si. Em relação aos coeficientes alfa de Cronbach, adotou-se a classificação de Murphy e Davidsholder (1988).

\section{Resultados e Discussão}

Com o objetivo de buscar evidências de validade com base na estrutura interna, realizou-se a análise fatorial exploratória do instrumento para verificação empírica dos seis fatores propostos por Holland (1975). Inicialmente, calculou-se a medida de adequação da amostragem por meio do teste de Kayser-Meyr-Olklin (KMO) e teste de esfericidade de Bartlett, na qual foram encontrados resultados considerados bons, de acordo com Friel (2008), e que justificam a fatoração dos dados $(\mathrm{KMO}=0,83, p<0,01)$. Nesse sentido, realizou-se a análise fatorial exploratória com método de extração fatoração do eixo principal, rotação oblimin e tendo como critério para manutenção do item carga fatorial dos itens de 0,30 ou superior.

Assim, foram feitas algumas tentativas de caráter exploratório, sendo que 12 itens foram excluídos por não atingirem os critérios. Por fim, a melhor solução foi apresentada com um conjunto de 56 itens, que se agruparam em seis fatores, tal como esperado. Com essa quantidade de itens, testou-se novamente o modelo com seis fatores. Inicialmente calculou-se a adequação da amostra e foram encontrados resultados bons, havendo um aumento do KMO após a exclusão dos itens $(\mathrm{KMO}=0,86, p<0,01)$, justificando a fatoração, uma vez que foi rejeitada a hipótese nula de que a matriz dos dados é igual á matriz identidade (Damásio, 2012). Foram mantidos os mesmos critérios para a fatoração e os resultados indicaram seis fatores de modo mais consistente nos agrupamentos e explicando $60,23 \%$ da variância, valor considerado adequado para extração de fatores (Dancey \& Reid, 2013; Hair, Black, Babin, Anderson, \& Thatam, 2006). Todavia, alguns itens agruparam em fatores para os quais não foram elaborados inicialmente. A Tabela 1 apresenta os resultados da análise fatorial exploratória sendo que as letras antes dos itens se referem às tipologias para as quais eles foram construídos inicialmente e endossados pelos juízes.

Em relação à estrutura fatorial, foi possível corroborar os seis fatores teóricos propostos por Holland (1975), tal como em outros estudos que utilizaram a teoria como base para construção de instrumentos (Holland et al., 1994; Mansão \& Noronha, 2011; Mansão \& Yoshida, 2006; Sampaio, 2013). Todavia, criaram-se categorias agrupando itens que não foram construídos originalmente para a tipologia, com exceção do tipo Artístico, no qual todos os itens reunidos foram elaborados inicialmente para esse fator. Importante ressaltar que a análise fatorial fornece elementos estatísticos que precisam ser contextualizados com os elementos teóricos para decidir sobre a manutenção ou exclusão de itens (Figueiredo-Filho \& Silva-Junior, 2010). Nesse sentido, a análise qualitativa dos itens também precisa ocorrer após a coleta dos dados para identificar e compreender os motivos de diferenças encontradas (Urbina, 2007). A seguir, cada agrupamento sugerido pela análise fatorial exploratória será discutido, porém, ressalta-se que não há, na literatura, estudos que buscaram construir instrumentos de interesses profissionais para pessoas com deficiência visual ou que verificaram o funcionamento do construto na população em questão, fato que demandou em buscar explicações dos resultados na própria teoria de Holland e em estudos sobre formação subjetiva de personalidade e conceitos em pessoas com deficiência visual.

A tipologia Empreendedor reuniu 13 itens, sendo que nove foram elaborados originalmente para esse perfil. Quatro itens construídos para outras tipologias agruparam nesse fator, sendo dois do tipo Convencional (64 - Conferir os livros de balanço de uma empresa e 58 Gerenciar a administração de um hotel) e dois do tipo Social (44 - Participar da elaboração de políticas públicas e 45 - Intermediar o diálogo entre pessoas que não falam a mesma língua). Em relação aos itens 64 e 58, embora 
tenham sido criados para representar atividades sistemáticas e burocráticas características do tipo Convencional, também envolvem as questões financeiras ou de liderança que são aspectos relacionados ao perfil Empreendedor, conceitualizado como aquele que reúne pessoas que preferem atividades nas quais encontrem a valorização dos aspectos econômicos e em que possam liderar pessoas (Holland et al., 1994).

Tabela 1

Fatores Obtidos na Análise Fatorial dos Itens da EIDV, Precisão e Variância Explicada

\begin{tabular}{|c|c|c|c|c|c|c|}
\hline \multirow{2}{*}{ Tipologia/Item } & \multicolumn{6}{|c|}{ Fatores } \\
\hline & $E$ & A & $\mathrm{S}$ & I & $\mathrm{C}$ & $\mathrm{R}$ \\
\hline E - Gerenciar planos de carreira... & 0,70 & & & & & \\
\hline E - Planejar aplicações financeiras... & 0,70 & & & & & \\
\hline E - Definir estratégias de criação de... & 0,66 & & & & & \\
\hline E - Intermediar a compra de... & 0,65 & & & & & \\
\hline E - Auxiliar a equipe a alcançar... & 0,64 & & & & & \\
\hline E - Negociar preços com clientes & 0,62 & & & & & \\
\hline E - Aconselhar investimentos... & 0,51 & & & & & \\
\hline E - Gerenciar a administração de... & 0,46 & 0,35 & & & & \\
\hline E - Vender produtos ou serviços... & 0,45 & & & & & 0,40 \\
\hline E - Fazer um curso de vendas & 0,43 & & & & & 0,39 \\
\hline C - Conferir livros de balanço de... & 0,41 & & & & & 0,31 \\
\hline S - Participar da elaboração de... & 0,34 & & & 0,31 & & \\
\hline S - Intermediar o diálogo entre... & 0,32 & & & & & \\
\hline A - Criar blocos ou programas para... & & 0,75 & & & & \\
\hline A - Participar da produção de um... & & 0,73 & & & & \\
\hline A - Fazer a locução de notícias em... & & 0,73 & & & & \\
\hline A - Atuar em uma peça teatral & & 0,69 & & & & \\
\hline A - Elaborar a lista de música de... & & 0,69 & & & & \\
\hline A - Apresentar um programa de... & & 0,68 & & & & \\
\hline A - Criar montagens de músicas para.. & & 0,61 & & & & \\
\hline A - Compor uma música a partir de... & & 0,59 & & & & \\
\hline A - Dançar para um grande público & & 0,49 & & & & \\
\hline S - Auxiliar professores a... & & & $-0,79$ & & & \\
\hline S - Orientar professores para... & & & $-0,74$ & & & \\
\hline S - Realizar psicoterapia com... & & & $-0,68$ & & & \\
\hline S - Propor estratégias de... & & & $-0,63$ & & & \\
\hline S - Desenvolver materiais didáticos... & & & $-0,60$ & & & \\
\hline S - Executar atividades para... & & & $-0,60$ & & & 0,35 \\
\hline S - Analisar os conflitos sociais de... & 0,31 & & $-0,59$ & & & \\
\hline I - Planejar um cardápio saudável... & & & $-0,34$ & & & 0,55 \\
\hline S - Dar aula para alunos de.... & & & $-0,31$ & & & \\
\hline A - Idealizar a organização de uma... & & & $-0,31$ & & & \\
\hline I - Escrever artigos científicos & & & & 0,55 & & \\
\hline I - Pesquisar profundamente sobre... & & & & 0,52 & & \\
\hline I - Ler artigos de revistas científicas & & & & 0,51 & & \\
\hline R - Analisar os dados numéricos de... & 0,35 & & & 0,42 & & \\
\hline R - Montar e gerenciar os bancos de... & 0,32 & & & 0,41 & & \\
\hline C - Controlar os dados de arquivos... & 0,31 & & & 0,36 & & \\
\hline R - Configurar redes de... & & & & & 0,88 & \\
\hline R - Programar sistemas... & & & & & 0,84 & \\
\hline C - Calcular a folha de pagamentos... & 0,50 & & & & 0,32 & \\
\hline C - Fiscalizar o funcionamento de... & & & & & 0,76 & \\
\hline C - Registrar a movimentação... & 0,67 & & & & 0,34 & \\
\hline
\end{tabular}


Tabela 1 (continuação)

Fatores Obtidos na Análise Fatorial dos Itens da EIDV, Precisão e Variância Explicada

\begin{tabular}{|c|c|c|c|c|c|c|}
\hline \multirow{2}{*}{ Tipologia/Item } & \multicolumn{6}{|c|}{ Fatores } \\
\hline & $E$ & A & $\mathrm{S}$ & I & $\mathrm{C}$ & $\mathrm{R}$ \\
\hline C - Traduzir livros para o... & & & & & 0,30 & \\
\hline $\mathrm{R}$ - Produzir massas para pães e bolos & & & & & & 0,70 \\
\hline R - Auxiliar na limpeza de um... & & & & & & 0,62 \\
\hline R - Preparar refeições em um... & & & & & & 0,58 \\
\hline R - Realizar chamadas telefônicas... & & & & & & 0,56 \\
\hline C - Operar elevadores para... & & & & & & 0,55 \\
\hline I - Prevenir e tratar lesões musculares & & & & & & 0,52 \\
\hline A - Pintar quadros em um ateliê & & & & & & 0,50 \\
\hline C - Catalogar livros de uma coleção & & & & & 0,42 & 0,47 \\
\hline A - Criar peças de artesanato para... & & & & & & 0,45 \\
\hline A - Escrever um livro de histórias & & 0,45 & & & & 0,32 \\
\hline A - Escrever poemas para um recital & & 0,33 & & & & 0,30 \\
\hline Alfa de Cronbach & 0,92 & 0,89 & 0,91 & 0,73 & 0,88 & 0,88 \\
\hline \% de variância explicada & 32,56 & 9,28 & 6,20 & 5,54 & 3,68 & 2,94 \\
\hline
\end{tabular}

Nota. $\mathrm{R}=$ Realista, I=Investigativo, A=Artístico, $\mathrm{S}=$ Social, E=Empreendedor, C=Convencional.

Os itens 44 e 45 elaborados para representar atividades relativas à ajuda ao próximo que é uma das principais características do perfil Social, também reúnem características do tipo Empreendedor como a articulação verbal. Assim, as atividades representadas nesses dois itens referem-se a questões que envolvem a capacidade de convencimento verbal, articulações políticas e de poder, sendo estes predicados marcantes na tipologia Empreendedor (Holland, 1985). Desse modo, optou-se por aceitar os quatro itens no fator Empreendedor, uma vez que reúnem atributos próprios da personalidade vocacional em questão.

$\mathrm{Na}$ tipologia Social formada pelo agrupamento de 10 itens na análise fatorial, dois itens foram construídos para outras dimensões. O item 17 (Planejar um cardápio saudável de refeições de uma escola) foi elaborado para o perfil Investigativo, tendo em vista que envolve uma atividade da área da saúde marcada pelas questões científicas, além do uso da imaginação para resolução de problema teórico. Todavia, a atividade retratada também envolve o cuidado e o bem-estar do próximo, característica do tipo Social (Holland, 1994) fazendo sentido mantê-lo nesse grupo. Outro item agrupado nesse fator foi elaborado para o perfil Artístico (34 - Idealizar a organização de uma sala), mas também se refere a uma atividade na qual está envolvido muito mais de convencionalismo/organização do que a desorganização própria do Artístico, sendo essa uma das características que Holland (1975) usa para diferenciar esse perfil do Social.

O perfil Investigativo agrupou a menor quantidade de itens, sendo que três agrupados não foram construídos para a tipologia. Os dois itens realistas caracterizados por atividades pouco sociáveis e ordenadas (1 - Analisar dados numéricos de um estudo e 2 - Montar e gerenciar o banco de dados de uma pesquisa) criados a partir da profissão de Estatístico, envolvem questões ligadas à área da pesquisa, aspecto concernente do perfil Investigativo (Holland, 1975). O item Convencional (65 - Controlar os dados de arquivos institucionais) pode ter se agrupado nesse item pela semelhança semântico dos demais itens, como manipulação de dados e organização de um conteúdo, envolvendo o contexto de pesquisa, gerando um padrão de respostas congruente.

Dos seis itens agrupados no tipo Convencional, dois eram listados inicialmente como Realista (3 - Configurar redes de computadores em uma empresa e 5 - Programar sistemas computacionais), todavia, a relação das tipologias no modelo hexagonal de Holland (1985) faz com que uma mesma atividade possa representar mais de um tipo de acordo com sua proximidade, caso dos perfis Realista e Convencional. Assim, é coerente a decisão de manter esses itens no fator Convencional, além do fato que dois outros itens do fator são relacionados a atividades de computação, o que pode ter criado uma congruência no padrão de respostas.

Por último, o tipo Realista foi o que mais reuniu itens elaborados para outras tipologias, sendo que, dos 11 itens agrupados, oito foram construídos para outros fatores. Em relação às atividades que normalmente são conceituadas como artísticas (23 - Criar peças de artesanatos para uma feira; 25 - Escrever um livro de história; 32 - Pintar quadros em um ateliê e 36 - Escrever poemas para um recital) embora demandem muito do processo criativo, estético e questões abstratas, para as pessoas com deficiência visual apresentaram-se como atividades concretas e manuais, sendo essas características predominantes no tipo Realista.

Nesse sentido, é importante repensar a percepção das pessoas com deficiência visual em relação às atividades mencionadas. $\mathrm{Na}$ ausência da visão para perceber 
cores, pintar e esculpir, esse público torna-se dependente dos outros sentidos, principalmente do tato. Assim, a própria concepção de beleza - característica muito valorizada no perfil artístico - para os deficientes visuais torna-se muito mais concreta e manual do que abstrata (Oliveira, 2002), desenvolvendo-se a partir do toque e não dos estímulos visuais. A mesma alteração ocorre para a escrita que, em videntes, têm maior influência pela forma visual das letras, em pessoas cegas, concentra-se no processo sensorial manual (Pagliuca, 1996). Embora esses novos arranjos de itens pareçam contradizer os conceitos teóricos do perfil, permitem reflexões acerca do desenvolvimento dos interesses profissionais de pessoas com deficiência visual e sobre a aplicabilidade da teoria de Holland para compreensão dos interesses dessa população.

A questão da habilidade manual como substituição das visuais também foi constatada nos outros quatro itens que agruparam na tipologia Realista. Assim, a atividade Investigativa (16 - Prevenir e tratar lesões musculares) e as convencionais (62 - Realizar chamadas telefônicas em uma empresa; 63 - Catalogar livros de uma coleção e 67 - Operar elevadores para transporte de pessoas e cargas), parecem ganhar mais ênfase no aspecto manual do que na investigação e organização, atributos das duas dimensões citadas. Assim, acredita-se que os pressupostos teóricos de Holland $(1975 ; 1985)$ para a conceitualização dessas atividades demandam de atualizações em relação ao desenvolvimento de habilidades, autopercepção e dos interesses profissionais de pessoas com deficiência visual, uma vez que as características próprias da deficiência modificam a compreensão das profissões e a categorização de profissões tradicionalmente alojadas em outras tipologias.
Posteriormente à análise fatorial exploratória, verificaram-se os índices de precisão por meio do cálculo do alfa de Cronbach para os fatores. No tipo Empreendedor, o alfa foi de valor 0,92 (elevado), porém observou-se que, com a exclusão dos itens 44 e 45, o coeficiente se manteria em $0,92 \mathrm{e}, \mathrm{com}$ isso, o fator se aproximaria dos demais em número de itens, desse modo, optou-se por excluí-los, ficando o tipo composto por 11 itens. Na tipologia Convencional, o coeficiente foi de 0,88 (moderado/elevado), mas com a exclusão do item 61 o índice passaria para 0,90 (elevado). Assim, houve a exclusão desse item, ficando o fator composto por cinco itens. As demais tipologias não tiveram itens excluídos e o menor valor foi encontrado na tipologia Investigativo ( $\alpha=0,73$; baixo). No tipo Social, o coeficiente foi considerado alto $(\alpha=0,91)$ e, nas tipologias Artístico e Realista, os valores foram médio/elevados ( $\alpha=0,89$ e 0,88 respectivamente). De modo geral, verifica-se que os seis fatores possuem boa confiabilidade para mensurar interesses profissionais.

Nesse sentido, após os estudos de consistência interna, foram excluídos 16 itens dos 68 itens selecionados a partir da análise de juízes e chegou-se a uma versão do instrumento com 52 itens, com a seguinte distribuição: Realista $(n=11)$, Investigativo $(n=6)$, Artístico $(n=9)$, Social $(n=10)$, Empreendedor $(n=11)$ e Convencional $(n=5)$. Considerando a nova organização dos itens nas tipologias, foram realizadas correlações de Pearson entre os escores formados pelos itens segundo a análise de juízes e o reagrupamento decorrente da análise fatorial. A Tabela 2 apresenta os resultados, sendo que as tipologias nas linhas se referem às elaboradas na análise de juízes, e as tipologias das colunas referem-se às resultantes da análise fatorial, sendo chamadas de tipologias empíricas.

Tabela 2

Correlações entre Fatores Teóricos e Empíricos

\begin{tabular}{ccccccc}
\hline & $\mathrm{R}(\mathrm{emp})$. & $\mathrm{I}(\mathrm{emp})$. & $\mathrm{A}(\mathrm{emp})$. & $\mathrm{S}$ (emp.) & $\mathrm{E}(\mathrm{emp})$. & $\mathrm{C}(\mathrm{emp})$. \\
\hline R & $0,75^{* *}$ & $0,60^{* *}$ & $0,38^{* *}$ & $0,55^{* *}$ & $0,67^{* *}$ & $0,72^{* *}$ \\
I & $0,79^{* *}$ & $0,63^{* *}$ & $0,51^{* *}$ & $0,75^{* *}$ & $0,64^{* *}$ & $0,47^{* *}$ \\
A & $0,70^{* *}$ & $0,30^{* *}$ & $0,96^{* *}$ & $0,57^{* *}$ & $0,49^{* *}$ & $0,22^{* *}$ \\
S & $0,64^{* *}$ & $0,52^{* *}$ & $0,49^{* *}$ & $0,96^{* *}$ & $0,62^{* *}$ & $0,37^{* *}$ \\
E & $0,60^{* *}$ & $0,51^{* *}$ & $0,44^{* *}$ & $0,59^{* *}$ & $0,98^{* *}$ & $0,68^{* *}$ \\
C & $0,72^{* *}$ & $0,59^{* *}$ & $0,39^{* *}$ & $0,56^{* *}$ & $0,83^{* *}$ & $0,82^{* *}$ \\
\hline
\end{tabular}

Nota. ${ }^{* *}$ Correlação é significativa no nível $p<0,0 . \mathrm{R}=$ Realista, I=Investigativo, A=Artístico, S=Social, E=Empreendedor, C=Convencional.

Todas as correlações entre as tipologias teóricas e as formadas empiricamente foram significativas e de magnitudes fortes (Dancey \& Reid, 2013), com destaque para as estabelecidas para os tipos Artístico, Social e Empreendedor consideradas quase perfeitas. Tal resultado pode estar relacionado com o fato de que essas tipologias concentram mais itens criados originalmente para elas em comparação com os demais. A correlação entre os escores teóricos e empíricos da tipologia Investigativo foi a que apresentou menor valor, fato que pode estar relacionado com o número de itens restante após a análise fatorial exploratória $(n=6)$ e pela quantidade de itens que carregaram nesse fator, mas que foram construídos originalmente e endossado pelos juízes como de outros tipos, 
ressaltando que esse fator também obteve menor índice de confiabilidade.

Assim, este estudo teve como objetivo apresentar a construção da EIDV e buscar evidências de validade baseadas no conteúdo e na estrutura interna como forma de responder a necessidade de avanços na área de avaliação psicológica, tornando-a mais acessível (Oliveira, 2013). Para além disso, as constantes transformações sociais no mundo do trabalho resultam na necessidade de atualizações no campo da Orientação Profissional (Duarte, 2000) em relação à formação dos orientadores, na compreensão dos sistemas de organização do trabalho e, também, dos instrumentais utilizados nos processos.

No processo de construção da EIDV, as avaliações das juízas pesquisadoras permitiram concluir que houve adequação teórica na formulação dos itens, o que levou a entender que haveria a replicabilidade do modelo de Holland. As avaliações das juízas psicólogas e os resultados do estudo piloto endossaram a acessibilidade do instrumento e adequação para o público-alvo, fato que pode ser constado na utilização de diversos recursos tecnológicos e assistivos para responder ao instrumento.

Em relação à análise de confiabilidade, os coeficientes obtiveram valores considerados adequados, indicando que as chances de erros na medida estão dentro do aceitável. A correlação entre os fatores teóricos endossados pelos juízes e entre os fatores formados empiricamente também apresentou valores elevados. Nesse sentido, há evidências de que, apesar do novo arranjo dos itens, os fatores continuam avaliando as mesmas características, sustentando a ideia de que essa nova configuração das atividades nas tipologias é adequada para compreender os interesses profissionais de pessoas com deficiência visual. Assim, atingiu-se o objetivo de buscar evidências de validade com base na estrutura interna, sendo confirmado empiricamente os seis fatores da teoria e com bons índices de precisão.

\section{Considerações Finais}

O estudo apresenta limitações na amostra tanto em número de participantes quanto na predominância de pessoas do estado de São Paulo quando comparados com outros estados. Todavia, houve uma grande dificuldade em acessar a população em questão, tanto por meio on-line como nas cinco instituições participantes. Outra limitação da amostra é em relação a faixa etária dos participantes constituída apenas por adultos e idosos. Assim, novos estudos devem ser realizados com adolescentes para verificar a adequação do uso da EIDV também nos processos de escolha profissional e com equilíbrio de participantes das diversas regiões do Brasil de modo a contemplar as variáveis culturais que podem interferir diretamente no nível e tipo de interesse profissional.

Foi possível testar a aplicabilidade do modelo teórico de Holland (1975) para a compreensão dos interesses profissionais de pessoas com deficiência visual. Verificouse, nos estudos de estrutura interna, que a compreensão de atividades profissionais por deficientes visuais não segue o modelo padrão teorizado pelo autor. Desse modo, ainda que os seis fatores tenham sido replicados, parece haver a necessidade de uma atualização da teoria para explicar o desenvolvimento dos interesses profissionais do público desta pesquisa, uma vez que estes foram impactados diretamente pela condição de deficiência. Assim, novas investigações devem ser realizadas para melhor compreensão da formação de interesses profissionais de pessoas com deficiência visual.

Ressalta-se, ainda, que as pesquisas com o público em questão parecem não ser pautas de investigações nos últimos anos, fato percebido pela ausência de literatura mais atualizada para discussão dos resultados. Espera-se que este estudo contribua para avanços na área de avaliação psicológica, de modo a permitir que públicos excluídos desse serviço possam utilizar de instrumentos que respeitem sua singularidade e especificidade. Para além disso, acredita-se que esta pesquisa contribua para a o campo de Orientação Profissional, tanto com a proposta de um instrumento como para reflexões acerca de como se dão os interesses profissionais de pessoas com deficiência visual. Muitos estudos com essa população ainda devem ser realizados nas duas áreas mencionadas, o que significa dizer que esta pesquisa não encerra a discussão sobre a temática, mas propõe-se a suscitar o interesse de outros pesquisadores de modo a avançar cada vez mais em serviços e instrumentos acessíveis, justos e igualitários.

\section{Referências}

Ambiel, R. A. M., Alves Lamas, K. C., \& Melo-Silva, L. L. (2016). Avaliação dos interesses profissionais no Brasil: Revisão da produção científica. Avaliação Psicológica, 15. doi: 10.15689/ap.2016.15ee.01

Ambiel, R. A. M., \& Polli, M. F. (2011). Análise da produção científica brasileira sobre avaliação psicológica em orientação profissional. Estudos Interdisciplinares em Psicologia, 2(1).103-121. Recuperado de http://pepsic.bvsalud.org/pdf/eip/v2n1/a08.pdf

American Psychological Association [APA]. (2011). Guidelines for Assessment of and Intervention with Persons with Disabilities. Recuperado de janeiro 2016, http://www.apa.org/pi/disability/resources/assessment-disabilities.aspx

Barnes, C. (2012). Re-thinking Disability, Work and Welfare. Sociology Compass, 6(6), 472-484. doi: 10.1111/j.1751-9020.2012.00464.x

Barros, L. O., \& Murgo, C. S. (2014). Orientação profissional e deficiência na produção científica nacional. Encontro: Revista de Psicologia, 17(26), 125-138. Recuperado de http://www.pgsskroton.com.br/seer/index.php/renc/article/view/2421/2321 
Campos, C. R., \& Nakano, T. C. (2014). Avaliação da inteligência de crianças deficientes visuais: proposta de instrumento. Psicologia: Ciência e profissão, 34(2), 406-419. doi: 10.1590/1982-3703000272013

Damásio, B. F. (2012). Uso da análise fatorial exploratória em psicologia. Avaliação psicológica, 11(2), 213-228. Recuperado de http://pepsic. bvsalud.org/pdf/avp/v11n2/v11n2a07.pdf

Dancey, C. P., \& Reidy, J. (2013). Estatística sem matemática para psicologia. (5 Ed). Porto Alegre: Penso.

Duarte, M. E. (2000). Da Orientação Profissional à Psicologia da Carreira: novos desafios. Organização E Sociedade, 6(17), 129-135. doi: 10.1590/S1984-92302000000100010

Figueiredo-Filho, D. B., \& Silva-Júnior, J. A. (2010). Visão além do alcance: Uma introdução à análise fatorial. Opinião Pública, $16(1), 160-185$. Recuperado de http://www.scielo.br/pdf/op/v16n1/a07v16n1

Filgueiras, A., Mendonça, I., Fontenele, B., Fioravanti-Bastos, A. C. M., Poyares, M., Oliveira, C. ... Marques, C. V. M. (2013). Características psicométricas da Escala de Stress Infantil (ESI) para avaliação do stress em crianças cegas. Psicologia Argumento, 31(75), 607-614. doi: 10.7213/psicol.argum.31.075.DS03

Friel, C. M. (2008). Notes on Factor Analysis. Sam Houston State University; Criminal Justice Centre.

Hair, J., Black, W. C., Babin, B. J., Anderson, R. E., \& Thatam, R. L. (2006). Multivariate Data Analysis. 6 a edição. Upper Saddle River, NJ: Pearson Prentice Hall.

Holland, J. L. (1975). Técnica de la elección vocacional: Tipos de personalidade y modelos ambientales. México: Editorial México.

Holland, J. L. (1985). Making Vocational Choices. A theory of vocational personalities \& work environments (2a . ed). Toronto: Prentice-Hall. (Original publicado em 1973).

Holland, J. L., Fritzsche, B. A., \& Powell, A. B. (1994). SDS-Self-Directed Search. Los Angeles, California: PAR- Psychological Assessment Resources.

Instituto Brasileiro de Geografia e Estatísticas - IBGE (2010). Censo Demográfico 2010: Características gerais da população, religião e pessoas com deficiência. Ministério do Planejamento, Orçamento e Gestão. Recuperado de http://biblioteca.ibge.gov.br/visualizacao/periodicos/94/ cd_2010_religiao_deficiencia.pdf

Lins, M. R. C. (2016). Relações entre a inteligência geral e a inteligência emocional: O papel do autoconceito em crianças e adolescentes videntes e com deficiência visual (Tese de doutorado). Universidade de Brasília.

Mansão, C. S. M., \& Noronha, A. P. P. (2011). Avaliação dos Tipos Profissionais de Holland: verificação da estrutura interna. Revista de Psícologia Trujillo, 13(1), 46-58. Recuperado de http://revistas.ucv.edu.pe/index.php/R PSI/article/view/308/197

Mansão, C. S. M., \& Yoshida, E. M. P. (2006). SDS - Questionário de busca autodirigida: precisão e validade. Revista Brasileira de Orientação Profissional, 7(2), 67-79. Recuperado de http://pepsic.bvsalud.org/pdf/rbop/v7n2/v7n2a07.pdf

Murphy, K. R., \& Davidshofer, C. O. (1988). Psychological testing: Principles and applications. Englewood Cliffs, New Jersey: Prentice Hall.

Oliveira, C. M. (2013). Evidências de validade de uma bateria informatizada para avaliação da personalidade adaptada ao Desenho Universal (Dissertação de mestrado). Centro de Filosofia e Ciências Humanas, Universidade Federal de Santa Catarina, Florianópolis.

Oliveira, J. V. G. (2002). Do essencial invisível: Arte e beleza entre os cegos. Rio de Janeiro: Revan/FAPERJ.

Pagliuca, L. M. F. (1996). A arte da comunicação na ponta dos dedos: A pessoa cega. Revista Latino-Americana de Enfermagem, 4(spe), $127-138$. doi: 10.1590/S0104-11691996000700013

Rebouças, C. B. D. A., Araújo, M. M. D., Braga, F. C., Fernandes, G. T., \& Costa, S. C. (2016). Avaliação da qualidade de vida de deficientes visuais. Revista Brasileira de Enfermagem, 69(1), 72-78.

Ribeiro, M. A. (2014). Orientação de carreira para jovens vivendo com sofrimento mental: Possibilidades e limites. Revista Psicologia Organizações e Trabalho, 14(4), 428-440. Recuperado de http://submission-pepsic.scielo.br/index.php/rpot/index

Salgado, F. (2002). O Balanço dinâmico e o papel da orientação profissional na elaboração de programas de inserção social de pessoas com deficiência. Análise Psicológica, 20(3), 407-418. Recuperado de http://www.scielo.mec.pt/pdf/aps/v20n3/v20n3a15.pdf

Savickas, M. L. (2015). Life-Design Counseling Manual. Rootstown, OH: Author.

Serra, B. D. M., Franzini Junior, C. A. A., Oliveira, E. V. D., Prado, M. T. A., Pacagnelli, F. L., Silva, R. C. R., \& Fernani, D. C. G. L. (2014). Avaliação psicomotora em crianças com deficiência visual. Colloquium Vitae, 5(1), 01-08.

Teixeira, M. A. P., Castro, G. D., \& Cavalheiro, C. V. (2008). Escalas de Interesses Vocacionais (EIV): Construção, validade fatorial e consistência interna. Psicologia em Estudo, 13(1), 179-186. Recuperado de http://www.scielo.br/pdf/pe/v13n1/v13n1a20.pdf

Urbina, S. (2007). Fundamentos da testagem psicológica. Tradução Claudia Dornelles. Porto Alegre: Artmed.

World Health Organization [WHO]. (2001). International Classification of functioning, disability and health: ICF. World Health Organization.

World Health Organization [WHO]. (2010). Action Plan for the Prevention of Avoidable Blindness and Visual Impairment - 2009-2013. Recuperado de http://www.who.int/blindness/ ACTION_PLAN_WHA62-1-English.pdf.

World Health Organization [WHO]. (2012). Relatório Muñdial sobre a deficiência (Lexicus Serviços Linguísticos, Trad.). São Paulo: Secretaria dos Direitos da Pessoa com Deficiência.

\section{Sobre os autores}

Leonardo de Oliveira Barros é psicólogo, mestre e doutorando em Psicologia com ênfase em Avaliação Psicológica pelo Programa de Pós-Graduação Stricto Sensu em Psicologia da Universidade São Francisco (bolsista CAPES).

Rodolfo. A. M. Ambiel é doutor em Psicologia e docente do Programa de Pós-Graduação Stricto Sensu em Psicologia da Universidade São Francisco. 